INPLASY

PROTOCOL

To cite: Cai et al. Insulin resistance in women with recurrent miscarriage: a systematic review and metaanalysis. Inplasy protocol 2021110055. doi:

10.37766/inplasy2021.11.0055

Received: 16 November 2021

Published: 16 November 2021

Corresponding author:

Wangyu Cai

1585322398@qq.com

Author Affiliation:

Zhejiang University School of Medicine.

Support: None.

Review Stage at time of this submission: Data analysis.

Conflicts of interest:

None declared.

\section{Insulin resistance in women with recurrent miscarriage: a systematic review and meta-analysis}

Cai, $\mathrm{W}^{1} ; \mathrm{Xu}, \mathrm{J}^{2}$.

Review question / Objective: This review aimed to investigate the association of insulin resistance in women with recurrent pregnancy loss compared to women with normal pregnancy history.

Condition being studied: Recurrent pregnancy loss.

Eligibility criteria: Observational studies that compared IR parameters in RPL patients to control women with normal pregnancy history were included. IR parameters included fasting blood glucose (FBG), fasting insulin (FIN), homeostasis model assessment for insulin resistance (HOMA-IR), glucose to insulin ratio (GI ratio). Only studies that were published in English were included. Review articles, opinions, book chapters, letters, published abstracts, animal studies, case reports, and studies with no suitable control group were excluded.

INPLASY registration number: This protocol was registered with the International Platform of Registered Systematic Review and Meta-Analysis Protocols (INPLASY) on 16 November 2021 and was last updated on 16 November 2021 (registration number INPLASY2021110055).

\section{INTRODUCTION}

Review question / Objective: This review aimed to investigate the association of insulin resistance in women with recurrent pregnancy loss compared to women with normal pregnancy history.
Condition being studied: Recurrent pregnancy loss.

\section{METHODS}

Participant or population: Women with recurrent pregnancy loss. 
Intervention: None.

Comparator: Women with normal pregnancy history.

Study designs to be included: Observational studies.

Eligibility criteria: Observational studies that compared IR parameters in RPL patients to control women with normal pregnancy history were included. IR parameters included fasting blood glucose (FBG), fasting insulin (FIN), homeostasis model assessment for insulin resistance (HOMA-IR), glucose to insulin ratio (GI ratio). Only studies that were published in English were included. Review articles, opinions, book chapters, letters, published abstracts, animal studies, case reports, and studies with no suitable control group were excluded.

Information sources: PubMed, Embase, and Web of Science.

Main outcome(s): Fasting blood glucose (FBG), fasting insulin (FIN), homeostasis model assessment for insulin resistance (HOMA-IR), glucose to insulin ratio (GI ratio).

Quality assessment / Risk of bias analysis: Newcastle-Ottawa scale.

Strategy of data synthesis: The mean difference (MD) or odds ratio (OR) with 95\% confidence interval $(\mathrm{Cl})$ were pooled to measure the effect size.

Subgroup analysis: To measure the effect of confounders on the effect size of potential moderators, subgroup analysis was performed.

Sensitivity analysis: To confirm the robustness of the results, a sensitivity analysis was performed by systematically excluding each study included in the analysis.
Keywords: recurrent miscarriage, insulin resistance, HOMA-IR, GI ratio, systematic review.

Contributions of each author:

Author 1 - Wangyu Cai.

Email: 1585322398@qq.com

Author 2 - Jian Xu.

Email: xuj@zju.edu.cn

Country(ies) involved: China. 